\title{
Allergy to polyethylene glycol has significant impact on daily life
}

\author{
Maria Bruusgaard-Mouritsen ${ }^{1}$, Jeanne Johansen², and Lene Garvey ${ }^{2}$ \\ ${ }^{1}$ Gentofte Hospital Skin and Allergy Department \\ ${ }^{2}$ Copenhagen University Hospital Gentofte
}

July 6, 2020

\begin{abstract}
Introduction: Polyethylene glycols (PEGs) are widely used as excipients in drugs, cosmetics and household products. Immediatetype allergy to PEGs including anaphylaxis are reported with low but increasing frequency. Low awareness of the allergenic potential of PEGs among consumers, manufacturers and doctors leads to under-diagnosis and under-reporting of PEG allergy, putting patients at risk of repeated severe reactions. The aims of this study were to investigate clinical manifestations, time to diagnosis and impact of a PEG allergy diagnosis on daily life of patients. Method: Ten PEG allergic patients answered a questionnaire about clinical manifestations, causes and impact on daily life of a PEG allergy, scored on a likert scale (0-10) before and after diagnosis. Results: Eight patients had experienced at least one anaphylactic reaction requiring adrenaline treatment. Anaphylaxis was caused by depot-steroids, antibiotic/analgesic tablets, antacids and laxatives. Seven patients reported repeated reactions before diagnosis (median 3, range 2-6). Median time from first reaction to diagnosis was 20 months (range 2-120). None of the patients experienced severe allergic reactions after the diagnosis. Median likert score of the impact on daily life before diagnosis was 7 compared to 4 after diagnosis. Conclusion: Daily life of PEG allergic patients is improved after diagnosis. Detailed information about the allergy, an allergy warning card, education in checking labels of new products, continued follow-up and advice from the Allergy Department were reported by patients to be important. Improved awareness about PEG allergy, clear product labelling and a standardized nomenclature is needed to improve care for these patients.
\end{abstract}

\section{Hosted file}

Complete Manuscript with Tables.pdf available at https://authorea.com/users/340035/articles/ 467173-allergy-to-polyethylene-glycol-has-significant-impact-on-daily-life 